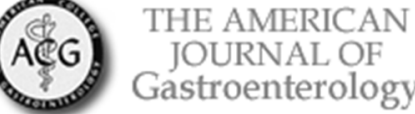

\title{
Genetic variants in PNPLA3 and TM6SF2 predispose to the development of hepatocellular carcinoma in individuals with alcohol-related cirrhosis
}

\begin{tabular}{|r|l|}
\hline Journal: & American Journal of Gastroenterology \\
\hline Manuscript ID & AJG-17-2309 \\
\hline Manuscript Type: & Original Contributions \\
\hline Keywords: & $\begin{array}{l}\text { Genetic Polymorphism, Alcoholic Liver Disease, Hepatocellular Carcinoma, } \\
\text { Risk Factors }\end{array}$ \\
\hline Manuscript Section: & Liver \\
\hline
\end{tabular}

\section{SCHOLARONE ${ }^{\text {m }}$ \\ Manuscripts}




\section{Genetic variants in PNPLA3 and TM6SF2 predispose to the development of hepatocellular carcinoma in individuals with alcohol-related cirrhosis}

Short title: Genetic risk for hepatocellular carcinoma

Felix Stickel $^{1^{*}}$, Stephan Buch ${ }^{2 *}$, Hans Dieter Nischalke ${ }^{3}$, Karl Heinz Weiss $^{4}$, Daniel Gotthardt ${ }^{4}$, Janett Fischer ${ }^{5}$, Jonas Rosendahl ${ }^{6}$, Astrid Marot ${ }^{7}$, Mona Elamly ${ }^{7}$, Markus Casper ${ }^{8}$, Frank Lammert $^{8}$, Andrew McQuillin ${ }^{9}$, Steffen Zopf ${ }^{10}$, Ulrich Spengler ${ }^{3}$, Silke Marhenke ${ }^{11}$, Martha Kirstein $^{11}$, Arndt Vogel ${ }^{11}$, Florian Eyer ${ }^{12}$, Johann von Felden ${ }^{13}$, Henning Wege ${ }^{13}$, Thorsten Buch $^{14}$, Clemens Schafmayer ${ }^{15}$, Felix Braun ${ }^{15}$, Pierre Deltenre ${ }^{7 \ddagger}$, Thomas Berg ${ }^{5 \ddagger}$, Marsha Y. Morgan $^{16 \ddagger}$, Jochen Hampe ${ }^{2 \ddagger}$

*FS and SB have contributed equally to the presented work and share premier authorship ${ }^{\dagger} \mathrm{PD}, \mathrm{TB}, \mathrm{MYM}$ and JH have contributed equally to the presented work and share senior authorship

${ }^{1}$ Department of Gastroenterology and Hepatology, University Hospital of Zurich, Switzerland

${ }^{2}$ Medical Department 1, University Hospital Dresden, TU Dresden, Germany

${ }^{3}$ Department of Internal Medicine I, University of Bonn, Germany

${ }^{4}$ Department of Internal Medicine IV, University Hospital Heidelberg, Heidelberg, Germany

${ }^{5}$ Hepatology Section, Division of Gastroenterology and Rheumatology, University Hospital Leipzig, Leipzig, Germany

${ }^{6}$ Department of Gastroenterology, University Hospital Halle/Saale, Germany

${ }^{7}$ Division of Gastroenterology and Hepatology, Centre Hospitalier Universitaire Vaudois, University of Lausanne, Switzerland

${ }^{8}$ Department of Medicine II, Saarland University Medical Center, Homburg, Germany

${ }^{9}$ Molecular Psychiatry Laboratory, Division of Psychiatry, University College London, UK

${ }^{10}$ Medical Department 1, University of Erlangen-Nuremberg, Germany

${ }^{11}$ Department of Gastroenterology, Hepatology and Endocrinology, Hannover Medical School, Germany

${ }^{12}$ Department of Clinical Toxicology, Klinikum rechts der Isar, Technical University of Munich, Germany

${ }^{13}$ First Department of Medicine, University Medical Center Hamburg-Eppendorf, Hamburg

${ }^{14}$ Institute of Laboratory Animal Science, University of Zurich, Schlieren, Switzerland

${ }^{15}$ Department of General and Thoracic Surgery, University Hospital Schleswig-Holstein, Campus Kiel, Germany

${ }^{16}$ UCL Institute for Liver \& Digestive Health, Division of Medicine, Royal Free Campus, University College London, United Kingdom 
Key words: candidate genes, genotyping, malignant transformation, host genetics, lipotoxicity, single nucleotide polymorphism

Word count: 3,182 (without title page, abstract, references, tables and figures)

Tables: 5

Figures: 1 (+3 supplementary figures $1 \mathrm{~A}-\mathrm{C}$ )

\section{Correspondence}

Felix Stickel, MD

Department of Gastroenterology and Hepatology, University Hospital Zürich, Rämistrasse 100, CH-8091 Zürich, Switzerland

Phone +41-31-338 7817

Fax +41-31-335 3519

Felix.stickel@uzh.ch

\section{Abbreviations:}

$\begin{array}{lll}\text { AUDIT } & - & \text { Alcohol Use Disorders Identification Test } \\ \text { BMI } & - & \text { body mass index } \\ \text { ELISA } & - & \text { enzyme-linked sorbent assay } \\ \text { GWAS } & - & \text { genome-wide association study } \\ \text { HCC } & - & \text { hepatocellular carcinoma } \\ \text { MAF } & - & \text { minor allele frequency } \\ \text { MBOAT7 } & - & \text { membrane bound O-acyltransferase domain containing } 7 \\ \text { NAFLD } & - & \text { non-alcoholic fatty liver disease } \\ \text { NCAN } & - & \text { neurocan } \\ \text { NPV } & - & \text { negative predictive value } \\ \text { OR } & - & \text { odds ratio } \\ \text { PAF } & - & \text { population-attributable fraction } \\ \text { PNPLA3 } & - & \text { patatin-like phospholipase domain-containing } 3 \\ \text { SNP } & - & \text { single nucleotide polymorphism } \\ \text { TM6SF2 } & - & \text { transmembrane } 6 \text { superfamily member } 2\end{array}$




\section{Funding}

This work was supported by grants from the Swiss National Funds (SNF no. 310030_169196) and the Swiss Foundation for Alcohol Research (SSA) to FS, and the Liver Systems Medicine (LiSyM) Network funded by the German Federal Ministry for Education and Research (BmBF) to JH. HDN and US were supported by a grant from the Deutsche Krebshilfe (70112169). 


\section{STUDY HIGHLIGHTS}

\section{What is current knowledge?}

- The gene variant PNPLA3 rs738409 is an established risk factor for the development of alcohol-related cirrhosis and for the subsequent development of hepatocellular carcinoma (HCC) within this population

- Two other risk variants, TM6SF2 rs58542926 and MBOAT7 rs641738, are associated with the development of alcohol-related cirrhosis but their role in the subsequent development of HCC is unclear

What is new here?

- While PNPLA3 rs738409 is confirmed as a risk factor for HCC in alcoholic cirrhotics, the present study identifies TM6SF2 rs58542926 as an independent risk factor for the development of HCC in people with alcohol-related cirrhosis

- The dual carriage of these risk variants in PNPLA3 and TM6SF2 accounts for almost $50 \%$ of the attributable risk for the development of HCC in this population

- In contrast to its role as a risk factor for alcoholic cirrhosis, MBOAT7 rs641738 does not seem to confer risk to HCC development in this risk group

- Genotype-based risk stratification for patients with alcohol-related cirrhosis may guide the development of rational, cost-effective surveillance programmes to facilitate early HCC detection 


\section{American Journal of Gastroenterology}

Stickel et al.

Genetic risk of hepatocellular carcinoma

\section{ABSTRACT}

Objectives: Variants in patatin-like phospholipase domain-containing 3 (PNPLA3; rs738409), transmembrane 6 superfamily member 2 (TM6SF2; rs58542926) and membrane bound Oacyltransferase domain containing 7 (MBOAT7; rs641738) are risk factors for the development of alcohol-related cirrhosis. Within this population, PNPLA3 rs738409 is also an established risk factor for the development of hepatocellular carcinoma (HCC). The aim of this study was to explore possible risk associations of TM6SF2 rs58542926 and MBOAT7 rs641738 with HCC.

Methods: Risk variants in PNPLA3, TM6SF2 and MBOAT7 were genotyped in 751 cases with alcohol-related cirrhosis and HCC and in 1165 controls with alcohol-related cirrhosis without HCC. Association with the risk of developing HCC was analyzed using multivariate logistic regression.

Results: The development of HCC was independently associated with PNPLA3 rs738409 $\left(\mathrm{OR}_{\text {adjusted }} 1.84[95 \% \mathrm{Cl} 1.55-2.18], \mathrm{p}=1.85 \times 10^{-12}\right)$ and TM6SF2 rs58542926 (OR adjusted 1.66 [1.30-2.13], $p=5.13 \times 10^{-05}$ ) using an additive model and after controlling for sex, age, body mass index and type 2 diabetes mellitus; the risk associated with carriage of MBOAT7 rs641738 (OR ${ }_{\text {adjusted }} 1.04$ [0.88-1.24], $\left.p=0.61\right)$ was not significant. The population-attributable fractions were $43.5 \%$ for PNPLA3 rs738409, $11.5 \%$ for TM6SF2 rs58542926, and $49.9 \%$ for carriage of both variants combined.

Conclusions: Carriage of TM6SF2 rs58542926 is an additional risk factor for the development of HCC in people with alcohol-related cirrhosis. Carriage of both PNPLA3 rs738409 and TM6SF2 rs58542926 accounts for half of the attributable risk for HCC in this population. Genotyping will allow for more precise HCC risk stratification of patients with alcohol-related cirrhosis, and genotype-guided screening algorithms would optimize patient care. 


\section{American Journal of Gastroenterology}

\section{INTRODUCTION}

The incidence of hepatocellular carcinoma (HCC) is increasing and it is now the fifth most frequent cancer and third most frequent cause of cancer-related mortality world-wide [1-3]. In Western countries, the presence of cirrhosis is a major risk factor for the development of HCC which only rarely evolves in non-cirrhotic livers [1,4,5]. Persistent high alcohol consumption is the leading cause of cirrhosis in these areas. The prognosis of patients with $\mathrm{HCC}$ is grave as it is often detected only when it reaches an advanced stage.

Between 5 to $15 \%$ of patients with alcohol-related cirrhosis are potentially at risk for developing HCC with a 5-year cumulative risk of around 8\% [1]. Identifying diseasemodulating factors which render some individuals more susceptible to its development than others is clearly of major importance as it would allow more specific targeting of surveillance programmes. Older age, male sex, and more advanced liver disease at presentation, are known to be important predictors but do not account for more than a small proportion of the observed risk variance. Mounting evidence suggest that host genetic factors may significantly modify the risk for developing HCC. Thus, identifying these factors in patients with alcoholrelated cirrhosis is clearly of major importance.

A single nucleotide polymorphism (SNP) rs738409 C>G in patatin-like phospholipase domaincontaining 3 (PNPLA3) is an established risk factor for the development of alcohol-related cirrhosis in Caucasian populations [6-9]. A recent meta-analysis of individual patient data from candidate gene studies has shown that the PNPLA3 rs738409 G allele is also an independent risk factor for the development of HCC in this patient population [10,11]. Recently, variants in two further genes viz. rs58542926 in transmembrane 6 superfamily member 2 (TM6SF2) and rs641738 in membrane bound O-acyltransferase domain containing 7 (MBOAT7) were identified as risk factors for the development of alcohol-related cirrhosis in a genome-wide 
association study (GWAS) [12]. Although a proportion of the cases included in the GWAS had developed HCC, the numbers were too small to allow for a meaningful subset analysis. However, data from two candidate gene studies support a role for variants in or linked to TM6SF2 as potential risk factors for HCC development. Thus, Nischalke et al. found a significant association between rs2228603 in the gene coding for neurocan (NCAN) and the development of HCC in people with alcohol-related cirrhosis [13]; this variant is in close linkage disequilibrium with TM6SF2 rs58542926. Falleti et al. genotyped TM6SF2 rs58542926 in 226 cases with alcohol-related cirrhosis of whom 75 (33\%) had HCC [14]. The rs58542926 $\mathrm{T} /$ *genotype was more frequent in those cases with HCC (adjusted odd ratio [OR] 2.57; $\mathrm{p}=0.035)$. They found no interaction between PNPLA3 rs738409 and TM6SF2 rs58542926 in relation to HCC risk. These preliminary findings need to be further explored. No studies have been undertaken, to date, to explore the possible risk association between MBOAT rs641738 and the development of HCC in patients with alcohol-related cirrhosis.

The aim of the present study was to determine whether variants in PNPLA3, TM6SF2 and MBOAT7 are risk factors for the development of HCC, alone or in synergy, in a large, wellcharacterized population of people with alcohol-related cirrhosis. 


\section{METHODS}

\section{Patient cohorts}

For purposes of this study cases were defined as people with alcohol-related cirrhosis and HCC and controls as people with alcohol-related cirrhosis but without evidence of HCC. Cases and controls were recruited from Hepatology centres across Europe (Switzerland, Germany, United Kingdom) and were of self-reported Swiss German/German/British/Irish ancestry.

The diagnosis of alcohol-related cirrhosis was established as described previously [12], and based on a history of prolonged, sustained alcohol intake of a minimum of $40 \mathrm{~g} / \mathrm{day}$, if female, and $60 \mathrm{~g} /$ day, if male, and histological examination of liver tissue; or compatible historical, clinical, laboratory, radiological and endoscopic features. Patients were excluded if they had any other potential cause of liver injury, although a proportion of the participants were overweight. The diagnosis of HCC was based on histological examination of tumour tissue or evidence on imaging, preferably using two modalities, of lesion that are hypervascular in the arterial phase with washout in the portal venous or delayed phases [15]. The severity of underlying alcohol-related liver injury was assessed using Pugh's modification of the Child's grading system [16].

\section{DNA preparation and genotyping}

DNA was prepared using the FlexiGene chemistry (Qiagen, Hilden, Germany). For preparation of genotyping, DNA samples were evaluated by gel electrophoresis and adjusted to 20-30 ng/ $\mu$ l DNA content using the Picogreen fluorescent dye (Molecular Probes - Invitrogen, Carlsbad, Ca, USA) on a robotic platform using TECAN liquid handling equipment. One microliter of genomic DNA was amplified with the GenomiPhi (Amersham, Uppsala, Sweden) whole genome amplification kit and fragmented at $99^{\circ} \mathrm{C}$ for three minutes. Five ng of DNA 
were dried overnight in TwinTec hardshell 384well plates (Eppendorf, Hamburg, Germany) at room temperature. Genotyping of PNPLA3 rs738409 (Assay ID HCV7241_10), TM6SF2 rs58542926 (Assay ID HCV89463510_10), and MBOAT7 rs641738 (Assay ID HCV8716820_10) was performed using TaqMan ${ }^{\circledR}$ SNP Genotyping Assays and chemistries (Applied Biosystems, Foster City, CA, USA) on an automated platform with TECAN Freedom EVO and 384well TEMO liquid handling robots (TECAN, Männedorf, Switzerland) as described before.[12]

All process data were logged and administered with a database-driven LIMS. Reactions were completed and read in a 7900 HT TaqMan sequence detector system (Applied Biosystems, Foster City, CA, USA). The amplification reaction was carried out with the TaqMan universal master mix. Thermal cycling conditions consisted of 1 cycle for 10 minutes at $95^{\circ} \mathrm{C}$, followed by 45 cycles for 15 seconds at $95^{\circ} \mathrm{C}$, and 45 cycles for 1 minute at $60^{\circ} \mathrm{C}$.

\section{Statistical analysis}

Statistical analyses of data were performed using SPSS v.24.0 (IBM Corp., Armonk, NY, USA). Comparisons of the frequencies of the genotypes at the three loci were performed on $3 \times 2$ contingency tables between cases and controls. Genotypic and allelic tests of association were performed using $\chi^{2}$ statistics or Fisher's exact tests as appropriate. For all tests, nominal p-values are reported. The association between SNPs and the risk of HCC was estimated by calculating beta coefficients and their standard errors using logistic regression models adjusted for age, sex, BMI and diabetic status, assuming log-additive genetic effects. The interactions between polymorphic loci were examined by testing allelic and genotypic interaction terms in the logistic regression models. Results are expressed as odds ratios (OR) and 95\% confidence intervals $(\mathrm{Cl})$. 
Population Attributable Fraction

The population-attributable fraction (PAF) provides an epidemiological estimate of the proportion of the disorder that is attributable to the risk factor. Thus, it is an estimate of how much lower the frequency of HCC would be in the patients with alcohol-related cirrhosis, if the risk genotype(s) were eliminated from the population.

The PAF was estimated for heterozygous and homozygous carriage using the formula:

$$
(x-1) / x
$$

where $x=(1-p) 2+2 p(1-p) O R 1+p 2 O R 2 ; p$ is the allele frequency in alcohol-related cirrhosis, and OR1 and OR2 are the ORs associated with hetero- and homozygosity adjusted by sex, age, body mass index (BMI) and type 2 diabetes mellitus, respectively.[17]

Combined PAF estimates were calculated based on the individual PAFs for each associated SNP, assuming no multiplicative interaction amongst the SNPs.[17]

\section{Ethics}

The study was approved by the ethics committees of the participating institutions; all included subjects provided written informed consent prior to inclusion into the study. 


\section{RESULTS}

The cases were significantly older than the controls; proportionately more were male, overweight and had comorbid type 2 diabetes mellitus (Table 1). However, the cases were significantly less likely to have decompensated liver disease (Table 1).

Genotyping was completed for all three SNPs with call rates greater than 95\%; there were significant differences in the genotyping call rates between cases and controls but the distributions of the missing data were random and not skewed. All markers followed HardyWeinberg equilibrium (cutoff HWE $P>0.05$ ) in both the cases and controls (Table 2).

Allelic and genotypic associations of PNPLA3 rs738409 with HCC in alcohol-related cirrhosis were highly significant $\left(P_{\text {allelic }}=3.64 \times 10^{-20}, P_{\text {geno }}=6.14 \times 10^{-18}\right)$. The odds ratio $(\mathrm{OR})$ for homozygous carriers of the risk allele PNPLA3 rs738409 G was 3.26 (95\% Cl: 2.50-4.25) compared to the risk in heterozygous carriers of $1.70(95 \% \mathrm{Cl}: 1.37-2.10)$. The allelic and genotypic associations of TM6SF2 rs58542926 with HCC in alcohol-related cirrhosis were also highly significant at $P_{\text {allelic }}=8.93 \times 10^{-7}$ and $P_{\text {genotypic }}=7.74371 \times 10^{-6}$, respectively. The OR for homozygous carriage of the risk allele TM6SF2 rs58542926 T was 3.32 (95\% Cl: 1.76-6.24), while the OR for heterozygous carriage was 1.46 (95\% Cl: 1.15-1.84). In contrast, neither heterozygous nor homozygous carriage of the MBOAT7 rs641738 T allele was associated with HCC risk; the allelic and genotypic levels of significance were $P_{\text {allelic }}=0.639$ and $P_{\text {genotypic }}=0.676$ respectively while corresponding ORs for homozygous and heterozygous carriage of the T allele were 1.07 (95\% Cl: 0.83-1.38) and 0.96 (95\% Cl: 0.77-1.20).

The minor allele frequencies (MAF) of all three loci in the controls in the present study were in line with those observed in the previous GWAS in alcohol-related cirrhosis [12], but the frequencies of PNPLA3 rs738409 G and TM6SF2 rs58542926 T were higher in the cases. The MAF for MBOAT7 rs641738 T was similar in cases and controls. 
The robustness of the association of PNPLA3 rs738409 and TM6SF2 rs58542926 and the development of HCC was confirmed after adjustment for other known risk factors viz. age, sex, BMI and type 2 diabetes mellitus (Table 3). The lack of association with MBOAT7 rs641738 did not change following adjustment for these confounders.

The association of PNPLA3 rs738409 and TM6SF2 rs58542926 with HCC was consistent across the separate cohorts from Switzerland, Germany, Britain and Ireland, as was the lack of association with regard to MBOAT7 rs641738 (Supplementary Figures 1A-C).

The PAF\% for PNPLA3 rs738409 was $43.5 \%$ and for TM6SF2 rs58542926 $11.4 \%$ yielding a combined PAF\% of both risk loci of $49.9 \%$ (Table 4 ).

The proportion of cases and controls who carried either no, one out of two, or both risk variants in PNPLA3 and TM6SF2 illustrated the aggregated risk due to co-carriage of risk loci (Figure 1). Thus, only $10 \%$ of the controls but approximately $20 \%$ of the cases carried both risk alleles. 


\section{DISCUSSION}

Elucidating the genetic background of chronic liver diseases is important as it will enable an individual's risk to develop progressive liver damage to be assessed more precisely, and will allow greater insight into currently unknown molecular aspects of the underlying pathophysiology. Of particular importance is the definition of the risk factors predisposing an individual with an a priori cancer risk, i.e. cirrhosis, to the development of life-threatening malignancy particularly if defining the risk factors would allow development of a strategy for more individualized screening and targeted prevention.

The present study provides data on the largest cohort of patients with HCC on a background of alcohol-related cirrhosis undertaken to date and provides further confirmation that the PNPLA3 rs738409 $\mathrm{G}$ allele is the strongest independent genetic risk factor for HCC in this patient population $[10,11]$. The present analysis further expands the panel of genetic risk factors for HCC to include TM6SF2 rs58542926, albeit with lesser effect as reflected by its lower OR and PAF\%. It also showed a lack of association with MBOAT7 rs641738. There is only limited information on the possible risks associated with carriage of TM6SF2 rs58542926 and MBOAT7 rs641738 and the development of HCC with which to compare the findings of the present study.

Nischalke et al. in a relatively small, multicentre study found a significant risk association between carriage of rs2228603 in neurocan (NCAN) and the development of HCC in patients with alcohol-related cirrhosis [13]. This genetic variant had been linked the development of hepatic steatosis in a previous GWAS in nonalcoholic fatty liver disease (NAFLD) [18]. Subsequent genetic fine mapping showed that NCAN rs2228603 lies within $50 \mathrm{~kb}$ and is in strong linkage disequilibrium with TM6SF2 rs58542926 [19]. A subsequent exome-wide 


\section{American Journal of Gastroenterology}

Stickel et al.

Genetic risk of hepatocellular carcinoma

analysis conclusively identified TM6SF2 rs58542926 as the more likely risk factor for progressive NAFLD in this genomic region [20]. Falleti et al. [14] analyzed a cohort of 511 patients with cirrhosis of mixed aetiology, of whom 101 had HCC, and found an association between the risk of developing HCC and carriage of the TM6SF2 rs58542926 T allele in the 226 patients with alcohol-related cirrhosis (Adjusted $O R=2.57 ; p=0.035$ ). They found no interaction between PNPLA3 rs738409 and TM6SF2 rs58542926 in relation to HCC risk.

A recent candidate gene association study from Italy and the UK investigated the association between variants within MBOAT7 and HCC in a mixed cohort of patients with NAFLD, chronic hepatitis $\mathrm{C}$ and alcohol-related liver disease the majority of whom did not have cirrhosis [21]. The authors reported a significant association between MBOAT7 rs641738 and HCC in noncirrhotic patients with NAFLD, but only in the Italian cohort. The findings were not however replicated in a validation cohort of UK patients. The reasons for this discrepancy likely relates to the fact that development of HCC in people with non-cirrhotic alcohol- and viral-related liver disease in Western countries is very rare and hence the number of cases was small. Thus, the inability of these workers to replicate their findings may reflect the inconsistent of the chosen phenotypes studied and/or low statistical power.

The lack of association between HCC risk and MBOATrs641738 is further supported by a recent candidate gene study in people with chronic hepatitis $C$ which demonstrated that the influence of this variant is restricted to the transition from absence of fibrosis to mild fibrosis [22]. In this same study, no difference was observed in the MAF of MBOAT7rs641738 in a small subgroup of 75 people with HCV-related HCC within an entire cohort of 1,706 indicating a lack of statistical power. However, the present study was adequately powered to detect an allelic odds ratio of 1.18 with $80 \%$ power and hence the finding of no association between 
MBOAT7 rs641738 and the development of HCC in alcohol-related cirrhosis is robust [23] and was consistent across all study subpopulation (Supplementary Figure 1C).

In the present study carriage of risk alleles in PNPLA3 and TM6SF2 accounted for almost 50\% of the population-attributable genetic risk for the development of HCC in patients with alcohol-related cirrhosis. The combined PAF\% is usually lower than the sum of each individual PAR\% but not in this instance. The odds ratio was, in fact, greater than 3 for homozygous carriage and $\sim 1.5$ for heterozygous carriage for both variants, and hence surprisingly high for a germline genetic risk factor in a complex disorder.

The possibility that these genetic markers could be included in a stratification score to identify patients at high risk for HCC has to be considered. Hypothetically, screening intervals could be tailored according to the genetic signature in a given patient, applying longer intervals in those minimal inherited predisposition, and shorter periods for those who carry both risk alleles. However, any such proposals would need to be tested in prospective surveillance studies designed to assess possible patient benefit.

Anstee et al. [24] have recently looked at the clinical utility of PNPLA3 genotyping for stratifying the risk for developing HCC in patients with NAFLD in a reanalysis of the data published by Liu et al. [25]. They found that use of PNPLA3 genotyping alone to positively predict the risk of developing HCC was unlikely to be tenable. However, the negative predictive value (NPV) was substantially greater, suggesting that genotyping this SNP might have utility for selecting those individuals least likely to develop HCC and therefore least likely to benefit from surveillance.

Using a similar approach the diagnostic test performance for PNPLA3 and TM6SF2 genotyping for the detection of HCC risk in the present study cohort showed a NPV of $72.5 \%$ for the combination PNPLA3 rs738409 GG and TM6SF2 rs58542926 *T. Based on these figures, a 


\section{American Journal of Gastroenterology}

Stickel et al.

Genetic risk of hepatocellular carcinoma

schema for individual screening intervals is proposed with the caveat that any adoption must be subject to appropriate prospective evaluation (Table 5).

The distinct genetic profiles of the alcohol-related cirrhosis risk genes with regards to liver carcinogenesis also have intriguing functional implications. The PNPLA3 rs738409 GG variant is considered a loss-of-function mutation and as such is less able to hydrolyze triglycerides from lipid droplets leading to lipid trapping in hepatocytes and increased lipotoxicity. Since progression of fibrosis is only weakly associated with the degree of steatosis [26], additional effects beyond mere lipid accumulation are suspected. Thus, PNPLA3 rs738409 has been functionally linked to increased levels of circulating pro-inflammatory mediator intracellular adhesion molecule 1 (ICAM-1) [27], which is also overexpressed in human HCC stem cells and circulating HCC tumor cells [28]. Furthermore, in patients with NAFLD PNPLA3rs738409 G allele was found to be associated with decreased levels of adiponectin which has important anti-inflammatory, anti-fibrotic and anti-carcinogenic properties [29]. Recent data in cell culture and mice xenografts also suggest an effect of PNPLA3 rs738409 on the activation of the interleukin (IL)-6-Janus kinase (JAK2)/Signal Transducers and Activators of Transcription (STAT3) pathway via fatty acid-mediated inflammation [30]. Therefore, functional studies linking the 'lipid droplet disease' caused by PNPLA3 malfunction to cancer may likely unravel interesting insights into the pathophysiology of HCC.

While the promoting effect of PNPLA3 rs738409 on HCC evolution remains incompletely understood, even less is known about the possible role of genetic variation of TM6SF2 in this process. TM6SF2 is involved in the assembly and secretion of very low-density lipoproteins, similar to microsomal triglyceride transfer protein (MTTP), and thus a loss of function mutation would be compatible with lipid accumulation in hepatocytes. Accordingly, Sookoian et al. [31] performed allele-specific expression analyses of cDNA isolated from liver tissue 
from patients with NASH and confirmed that expression levels of the rs58542926 T allele are reduced to about $56 \%$ of that of the wild type $C$ allele. These findings suggest that carriage of the TM6SF2 T risk allele is associated with decreased hepatic gene and protein expression, leading to a loss of function similar to the mutant PNPLA3 variant. In turn, the wild type TM6SF2 protein has been associated with increased serum VLDL levels and an elevated cardiovascular risk [32], but less liver injury [33]. Whether and how there may be cross-talk between TM6SF2 and signaling pathways pertinent for HCC development beyond mere intracellular lipid accumulation and lipotoxicity remains to be elucidated.

This study has its limitations, primarily the fact that it was undertaken retrospectively and was restricted to Caucasian people with alcohol-related cirrhosis. Thus the findings do not necessarily define the genetic risks for HCC development in people with liver disease of other aetiologies or to other ethnic groups. However, it also has its strengths, including the size of the study population, and the fact similar effect sizes were observed across the component geographical cohorts. Thus, the presented data provide robust evidence for a strong effect of PNPLA3 rs738409 on HCC risk in patients with alcohol-related cirrhosis. In addition, TM6SF2 rs58542926 is identified and confirmed as a significant host genetic risk factor for HCC, while MBOAT7 rs641738 is not. In combination, PNPLA3 rs738409 and TM6SF2 rs58542926 account for almost $50 \%$ of the population-attributable risk for the development of HCC, and thus, should be evaluated further for stratification of patients at risk in screening programs. In addition, sight should not be lost of the fact that a significant proportion of the genetic variance still remains to be identified. 


\section{Acknowledgements}

The authors thank the Clinical Research Support Service of the CHUV-UNIL, Lausanne, Switzerland for providing the infrastructure for patient recruitment and collecting phenotypic data.

\section{Competing Interests}

None of the authors has any conflict of interest in relation to the contents of this manuscript. 


\section{REFERENCES}

1. Fattovich G, Stroffolini T, Zagni I, Donato F. Hepatocellular carcinoma in cirrhosis: incidence and risk factors. Gastroenterology 2004;127:S35-50.

2. McGlynn KA, London WT. The global epidemiology of hepatocellular carcinoma: present and future. Clin Liver Dis 2011;15:223-43.

3. Nordenstedt H, White DL, El-Serag HB. The changing pattern of epidemiology in hepatocellular carcinoma. Dig Liver Dis 2010;42(Suppl 3):S206-14.

4. Ascha MS, Hanouneh IA, Lopez R, et al. The incidence and risk factors of hepatocellular carcinoma in patients with nonalcoholic steatohepatitis. Hepatology 2010;51:1972-8.

5. Seitz HK, Stickel F. Molecular mechanisms of alcohol-mediated carcinogenesis. Nat Rev Cancer 2007;7:599-612.

6. Stickel F, Moreno C, Hampe J, Morgan MY. Genetics of alcohol dependence and alcoholrelated liver disease. J Hepatol 2017;66:195-211.

7. Chamorro AJ, Torres JL, Miron-Canelo JA, et al. Systematic review with meta-analysis: the I148M variant of patatin-like phospholipase domain-containing 3 gene (PNPLA3) is significantly associated with alcoholic liver cirrhosis. Aliment Pharmacol Ther 2014;40:57181.

8. Stickel F, Buch S, Lau K, et al. Genetic variation in the PNPLA3 gene is associated with alcoholic liver injury in Caucasians. Hepatology 2011;53:86-95.

9. Stickel F, Hampe J, Trepo E, et al. PNPLA3 genetic variation in alcoholic steatosis and liver disease progression. Hepatobiliary Surg Nutr 2015;4:152-60.

10. Singal AG, Manjunath H, Yopp AC, et al. The effect of PNPLA3 on fibrosis progression and development of hepatocellular carcinoma: a meta-analysis. Am J Gastroenterol 2014;109:325-34. 


\section{American Journal of Gastroenterology}

11. Trepo E, Nahon P, Bontempi G, et al. Association between the PNPLA3 (rs738409 C>G) variant and hepatocellular carcinoma: Evidence from a meta-analysis of individual participant data. Hepatology 2014;59:2170-7.

12. Buch S, Stickel F, Trepo E, et al. A genome-wide association study confirms PNPLA3 and identifies TM6SF2 and MBOAT7 as risk loci for alcohol-related cirrhosis. Nat Genet 2015;47:1443-8.

13. Nischalke HD, Lutz $\mathrm{P}$, Kramer $\mathrm{B}$, et al. A common polymorphism in the NCAN gene is associated with hepatocellular carcinoma in alcoholic liver disease. J Hepatol 2014;61:1073-9.

14. Falleti E, Cussigh A, Cmet S, et al. PNPLA3 rs738409 and TM6SF2 rs58542926 variants increase the risk of hepatocellular carcinoma in alcoholic cirrhosis. Dig Liver Dis 2016;48:69-75.

15. EASL-EORTC. Clinical Practice Guidelines: Management of hepatocellular carcinoma. J Hepatol 2012;56:908-43.

16. Pugh RN, Murray-Lyon IM, Dawson JL, et al. Transection of the oesophagus for bleeding oesophageal varices. BrJ Surg 1973;60:646-9.

17. Tomlinson IP, Webb E, Carvajal-Carmona L, et al. A genome-wide association study identifies colorectal cancer susceptibility loci on chromosomes 10p14 and 8q23.3. Nat Genet 2008;40:623-30.

18. Speliotes EK, Yerges-Armstrong LM, Wu J, et al. Genome-wide association analysis identifies variants associated with nonalcoholic fatty liver disease that have distinct effects on metabolic traits. PLoS Genet 2011;7:e1001324.

19. Liu YL, Reeves HL, Burt AD, et al. TM6SF2 rs58542926 influences hepatic fibrosis progression in patients with non-alcoholic fatty liver disease. Nat Commun 2014;5:4309. 
20. Kozlitina J, Smagris E, Stender S, et al. Exome-wide association study identifies a TM6SF2 variant that confers susceptibility to nonalcoholic fatty liver disease. Nat Genet 2014;46:352-6.

21. Donati B, Dongiovanni P, Romeo S, et al. MBOAT7 rs641738 variant and hepatocellular carcinoma in non-cirrhotic individuals. Sci Rep 2017;7:4492.

22. Thabet $K$, Asimakopoulos A, Shojaei M, et al. MBOAT7 rs641738 increases risk of liver inflammation and transition to fibrosis in chronic hepatitis C. Nat Commun 2016;7:12757.

23. Dupont WD, Plummer WD. Power and sample size calculations for studies involving linear regression. Control CliniTrials 1998;19:589-601.

24. Anstee QM, Liu YL, Day CP, Reeves HL. Reply to: HCC and liver disease risk in homozygous PNPLA3 p.I148M carriers approach monogenic inheritance. J Hepatol 2015;62:982-3.

25. Liu YL, Patman GL, Leathart JB, et al. Carriage of the PNPLA3 rs738409 C >G polymorphism confers an increased risk of non-alcoholic fatty liver disease associated hepatocellular carcinoma. J Hepatol 2014;61:75-81.

26. Valenti L, Al-Serri A, Daly AK, et al. Homozygosity for the patatin-like phospholipase3/adiponutrin I148M polymorphism influences liver fibrosis in patients with nonalcoholic fatty liver disease. Hepatology 2010;51:1209-17.

27. Pare G, Ridker PM, Rose L, et al. Genome-wide association analysis of soluble ICAM-1 concentration reveals novel associations at the NFKBIK, PNPLA3, RELA, and SH2B3 loci. PLoS Genet 2011;7:e1001374.

28. Liu S, Li N, Yu X, et al. Expression of intercellular adhesion molecule 1 by hepatocellular carcinoma stem cells and circulating tumor cells. Gastroenterology 2013;144:1031-41. 
29. Valenti L, Rametta R, Ruscica M, et al. The I148M PNPLA3 polymorphism influences serum adiponectin in patients with fatty liver and healthy controls. BMC Gastroenterol 2012;12:111.

30. Liu Z, Chen T, Lu X, et al. Overexpression of variant PNPLA3 gene at I148M position causes malignant transformation of hepatocytes via IL-6-JAK2/STAT3 pathway in low dose free fatty acid exposure: a laboratory investigation in vitro and in vivo. Am J Transl Res 2016;8:1319-38.

31. Sookoian S, Castaño GO, Scian R, et al. Genetic variation in transmembrane 6 superfamily member 2 and the risk of nonalcoholic fatty liver disease and histological disease severity. Hepatology 2015;61:515-25.

32. Holmen OL, Zhang H, Fan $\mathrm{Y}$, et al. Systematic evaluation of coding variation identifies a candidate causal variant in TM6SF2 influencing total cholesterol and myocardial infarction risk. Nat Genet 2014;46:345-51.

33. Sookoian S, Pirola CJ. Meta-analysis of the influence of TM6SF2 E167K variant on plasma concentration of aminotransferases across different populations and diverse liver phenotypes. Sci Rep 2016;6:27718. 


\section{LEGENDS TO FIGURES}

\section{Figure 1}

Proportion of cases with alcohol-related cirrhosis and HCC by carriage of the risk variants in PNPLA3 and TM6SF2.

\section{Supplementary figures}

\section{Figure 1A}

Forrest plot displaying a consistent association of PNPLA3 rs738409 with HCC across different national cohorts. The association is similar in all three cohorts.

\section{Figure 1B}

Forrest plot displaying association of TM6SF2 rs58542926 with HCC in different national cohorts. The association is similar in all three cohorts.

\section{Figure 1C}

Forrest plots ahowing no significant association between MBOAT7 rs641738 with HCC in any of the three cohorts. 
Table 1

Characteristics of cases and controls included in the study

\begin{tabular}{|c|c|c|c|c|}
\hline Variable & $\begin{array}{c}\text { Total } \\
(n=1916)\end{array}$ & $\begin{array}{c}\text { Cases } \\
(n=751)\end{array}$ & $\begin{array}{l}\text { Controls } \\
(n=1165)\end{array}$ & $\begin{array}{l}\text { Significance } \\
\text { (p) }\end{array}$ \\
\hline $\begin{array}{l}\text { Proportion male } \\
\text { (n: \%) }\end{array}$ & $1496(78)$ & $679(90)$ & $817(70)$ & $<0.00001$ \\
\hline Age (yr) & $58 \pm 11$ & $61 \pm 10$ & $55 \pm 10$ & $<0.00001$ \\
\hline BMI $\left(\mathrm{kg} / \mathrm{m}^{2}\right)$ & $26.6 \pm 4.7$ & $27.9 \pm 4.7$ & $25.9 \pm 4.6$ & $<0.00001$ \\
\hline $\begin{aligned} \text { BMI }(\%) \\
\quad<25 \\
25-30 \\
\geq 30\end{aligned}$ & $\begin{array}{l}36 \% \\
42 \% \\
21 \%\end{array}$ & $\begin{array}{l}26 \% \\
42 \% \\
32 \%\end{array}$ & $\begin{array}{l}43 \% \\
42 \% \\
15 \%\end{array}$ & $<0.00001$ \\
\hline $\begin{array}{l}\text { Child Pugh Grade [16] (\%) } \\
\qquad \begin{array}{l}\text { A } \\
\text { B } \\
\text { C }\end{array}\end{array}$ & $\begin{array}{l}35 \% \\
40 \% \\
26 \%\end{array}$ & $\begin{array}{l}48 \% \\
36 \% \\
16 \%\end{array}$ & $\begin{array}{l}27 \% \\
42 \% \\
31 \%\end{array}$ & $<0.00001$ \\
\hline $\begin{array}{l}\text { Type II diabetes mellitus } \\
\text { (n: \%) }\end{array}$ & $27(1.4)$ & $42(5.6)$ & $18(1.5)$ & $<0.00001$ \\
\hline
\end{tabular}

Data are reported as mean \pm standard deviation or as number (\%) 


\section{Table 2}

Genotypic and allelic frequencies of PNPLA3, TM6SF2 and MBOAT7 in people with alcohol-related cirrhosis with HCC (cases) and without (controls)

\begin{tabular}{|c|c|c|c|c|c|c|c|c|c|c|c|c|}
\hline SNP & $\begin{array}{c}\text { Total } \\
(n=1916)\end{array}$ & & & & & & & $\begin{array}{l}\text { Unadjusted } \\
\text { OR (Cl 95\%) }\end{array}$ & $\begin{array}{l}\text { Adjusted } \\
\text { OR (Cl 95\%) }\end{array}$ & $P$ trend & $\mathbf{P}_{\text {allelic }}$ & $\mathbf{P}_{\text {genotypic }}$ \\
\hline PNPLA3 & & CC & CG & GG & MAF & fCG (\%) & fGG (\%) & CG vs CC & GG vs CC & & & \\
\hline Cases & 751 & 205 & 346 & 200 & 0.497 & $46 \%$ & $27 \%$ & \multirow{2}{*}{$1.70[1.37-2.10]$} & \multirow{2}{*}{$3.26[2.50-4.25]$} & \multirow{2}{*}{$5.45 \mathrm{E}-19$} & \multirow{2}{*}{$3.64 \mathrm{E}-20$} & \multirow{2}{*}{$6.14 \mathrm{E}-18$} \\
\hline Controls & 1165 & 508 & 505 & 152 & 0.347 & $43 \%$ & $13 \%$ & & & & & \\
\hline TM6SF2 & & CC & CT & TT & MAF (\%) & fCT (\%) & fTT (\%) & CT vs TT & CC vs TT & & & \\
\hline Cases & 751 & 558 & 164 & 29 & 0.148 & $22 \%$ & $4 \%$ & \multirow[t]{2}{*}{$1.46[1.15-1.84]$} & \multirow[t]{2}{*}{$3.32[1.76-6.24]$} & \multirow[t]{2}{*}{$1.22 \mathrm{E}-06$} & \multirow[t]{2}{*}{ 8.93E-07 } & \multirow[t]{2}{*}{$7.74371 \mathrm{E}-06$} \\
\hline Controls & 1165 & 957 & 193 & 15 & 0.096 & $17 \%$ & $1 \%$ & & & & & \\
\hline MBOAT7 & & CC & CT & $\mathrm{TT}$ & MAF (\%) & fCT (\%) & fTT (\%) & CT vs TT & CC vs TT & & & \\
\hline Cases & 751 & 203 & 363 & 185 & 0.488 & $48 \%$ & $25 \%$ & \multirow{2}{*}{$0.96[0.77-1.20]$} & \multirow{2}{*}{$1.07[0.83-1.38]$} & \multirow{2}{*}{0.377} & \multirow{2}{*}{0.639} & \multirow{2}{*}{0.676} \\
\hline Controls & 1165 & 314 & 583 & 268 & 0.480 & $50 \%$ & $23 \%$ & & & & & \\
\hline
\end{tabular}

MAF, minor allele frequency; OR, odds ratio 
Table 3

Multivariate analysis of factors associated with hepatocellular carcinoma in people with alcohol-related cirrhosis

\begin{tabular}{|c|c|c|}
\hline SNP & *Adjusted OR (95\% Cl) & Significance (p) \\
\hline PNPLA3 rs738409:G & $1.84(1.55-2.18)$ & $1.85 \times 10^{-12}$ \\
\hline PNPLA3 rs738409:CG & $2.07[1.58-2.73]$ & $1.87 \times 10^{-07}$ \\
\hline PNPLA3 rs738409:GG & $3.26[2.31-4.60]$ & $1.87 \times 10^{-11}$ \\
\hline TM6SF2 rs58542926:T & $1.66(1.30-2.13)$ & $5.13 \times 10^{-05}$ \\
\hline TM6SF2 rs58542926:CT & $1.67[1.23-2.26]$ & $1.03 \times 10^{-03}$ \\
\hline TM6SF2 rs58542926:TT & $2.86[1.34-6.12]$ & $6.75 \times 10^{-03}$ \\
\hline MBOAT7 rs641738:T & $1.04(0.88-1.24)$ & 0.61 \\
\hline MBOAT7 rs641738:CT & $0.90[0.67-1.20]$ & .473 \\
\hline MBOAT7 rs641738:TT & $1.09[0.78-1.53]$ & .619 \\
\hline
\end{tabular}

SNP: single nucleotide polymorphism; OR: odds ratio; $\mathrm{Cl}$ : confidence interval

* adjusted for age, gender, BMI, type II diabetes mellitus. 


\section{Table 4}

Population-attributable fraction of each variant with regard to the genetic risk of HCC in alcohol-related cirrhosis alone, and in combination.

\begin{tabular}{|l|c|}
\hline Genetic variant & $\begin{array}{c}\text { *Population Attributable Fraction } \\
(\%)\end{array}$ \\
\hline PNPLA3 rs738409 & 43.5 \\
\hline TM6SF2 rs58542926 & 11.5 \\
\hline PNPLA3 + TM6SF2 (combined) & 49.9 \\
\hline
\end{tabular}

* Estimated for heterozygous and homozygous carriage in one equation. 
Table 5

Proposed screening intervals for HCC of people with alcohol-related cirrhosis based on the genetic signature

\begin{tabular}{|l|c|}
\hline Genetic background & Screening interval \\
\hline PNPLA3 rs738409 WT (CC), TM6SF2 rs58542926:CC & 24 months \\
\hline TM6SF2 rs58542926:T* and PNPLA3 rs738409 CC & 12 months \\
\hline TM6SF2 rs58542926:CC and PNPLA3 rs738409 GG & 6 months \\
\hline PNPLA3 rs738409 GG and TM6SF2 rs58542926:T* & 3 months \\
\hline
\end{tabular}


Figure 1

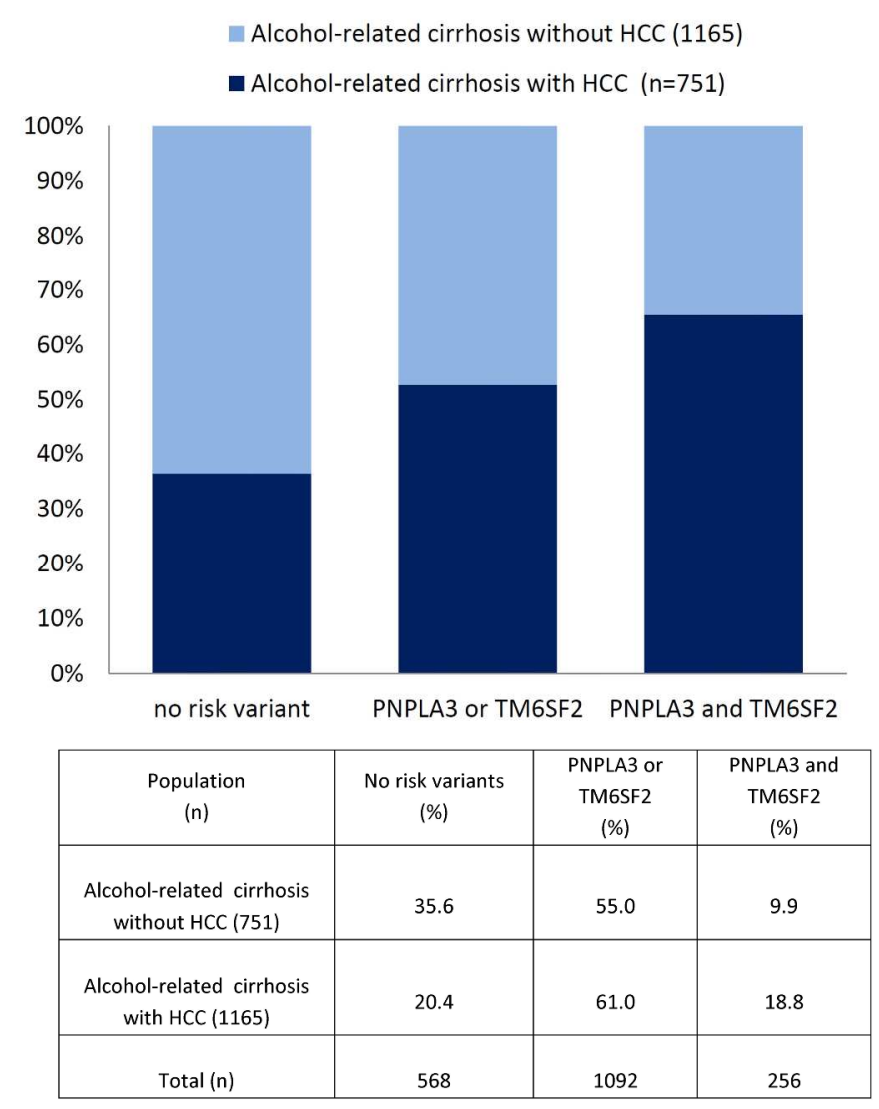

Proportion of cases with alcohol-related cirrhosis and HCC by carriage of the risk variants in PNPLA3 and TM6SF2

$297 \times 420 \mathrm{~mm}(300 \times 300 \mathrm{DPI})$ 
Association $p$-value $=4.99 \times 10^{-08}$

Heterogeneity $p$-value $=0.134$

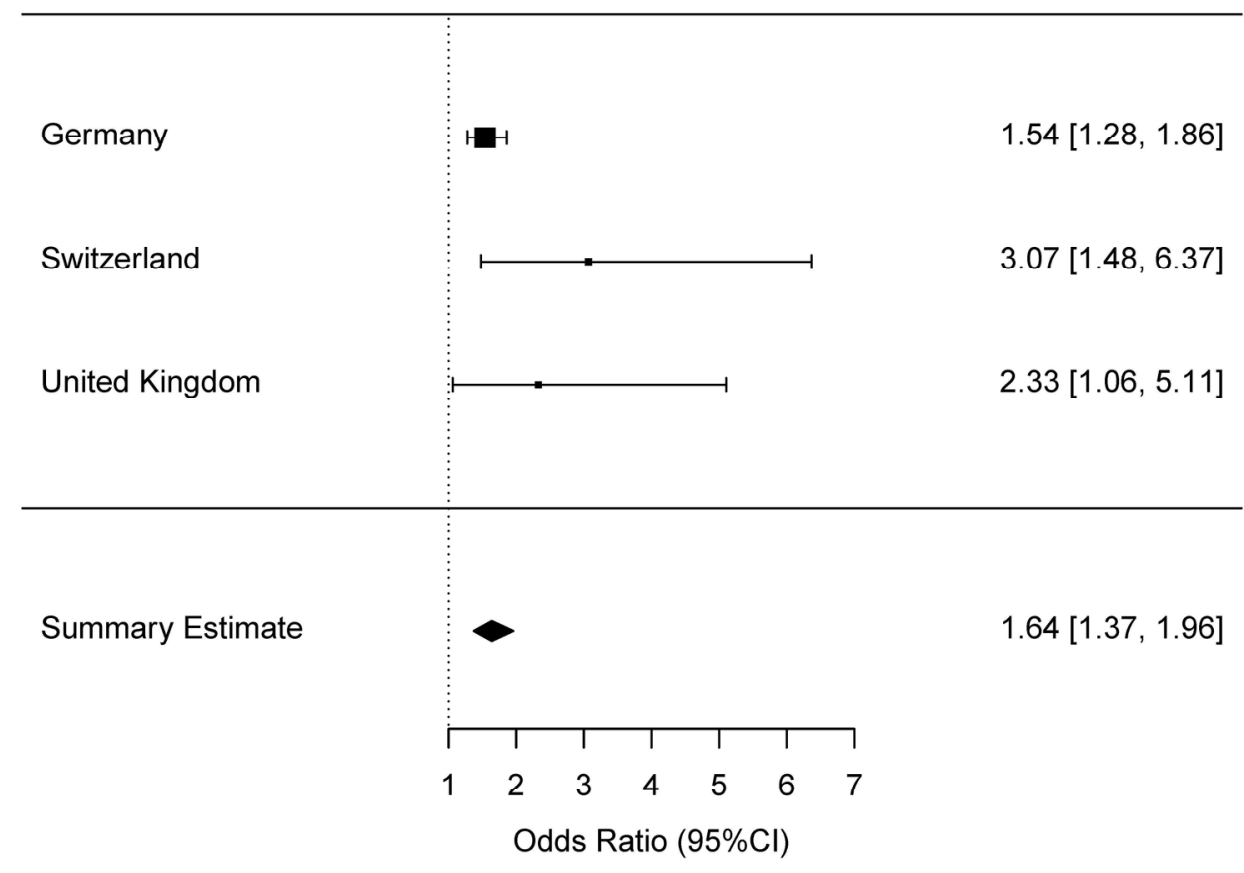

$177 \times 177 \mathrm{~mm}(300 \times 300$ DPI $)$ 
Association $p$-value $=2.47 \times 10^{-05}$

Heterogeneity $p$-value $=0.215$

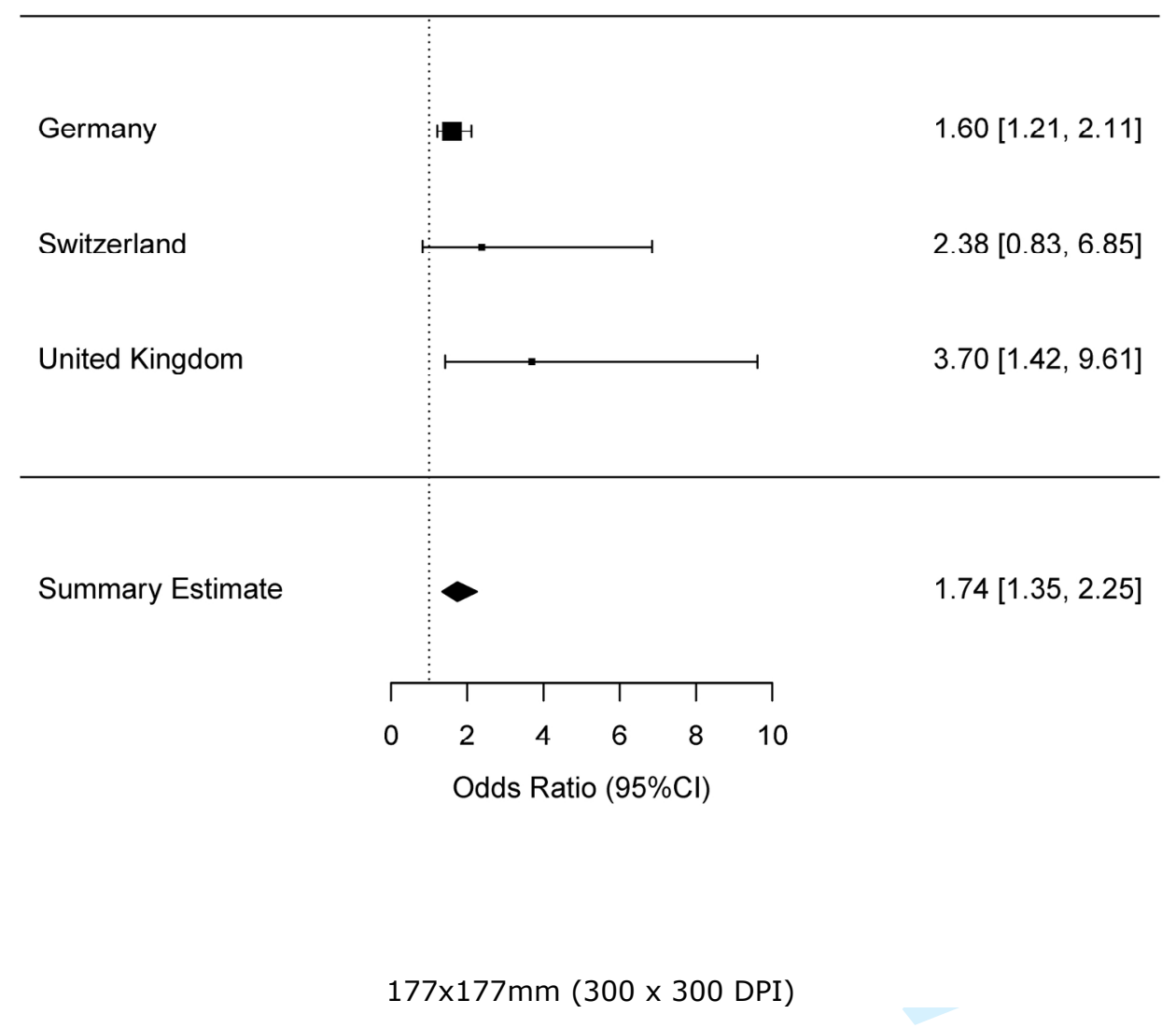


Association $p$-value $=0.532$

Heterogeneity $p$-value $=0.456$

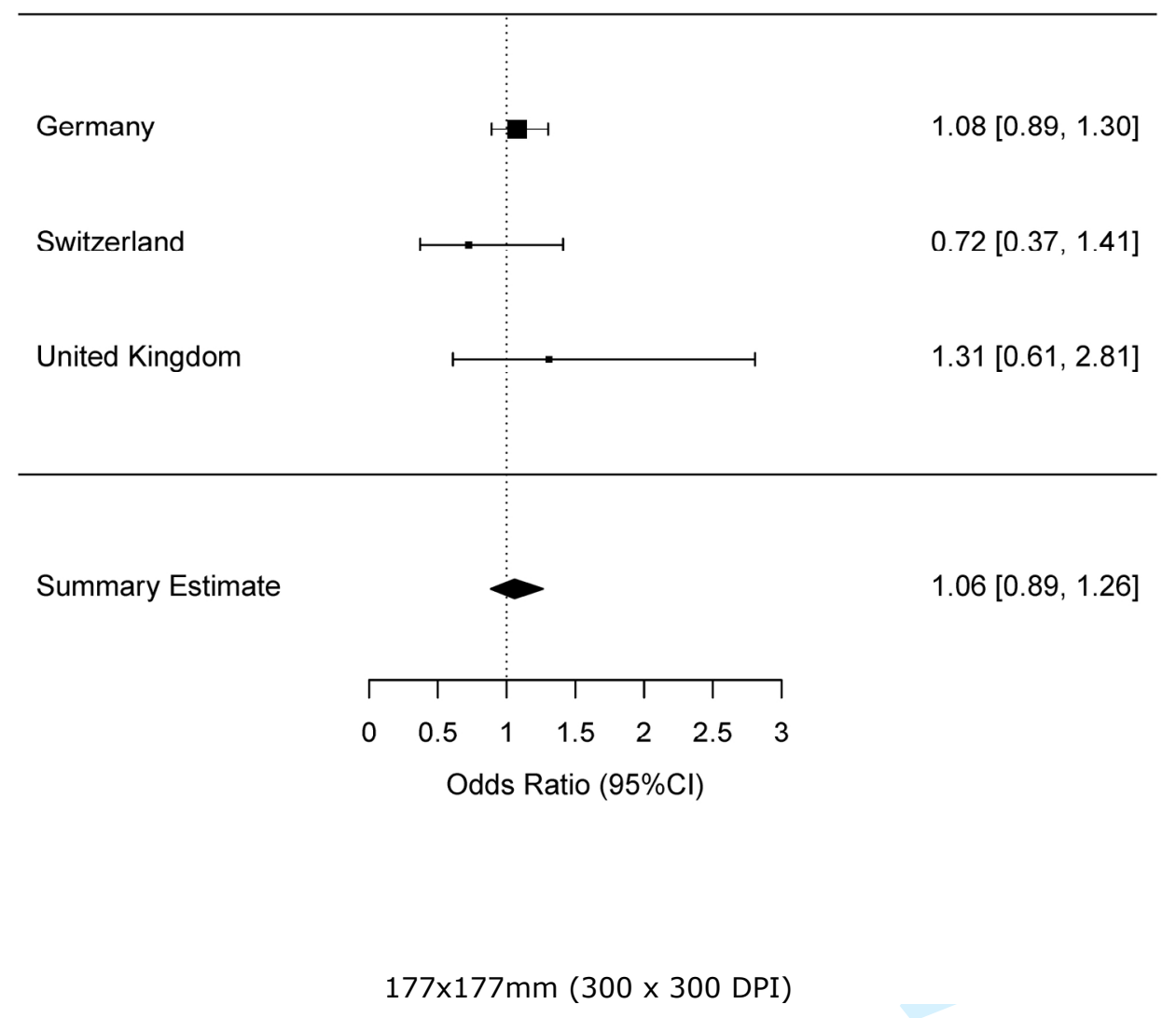

